EPJ Web of Conferences 38, 14005 (2012)

DOI: $10.1051 /$ epjconf/20123814005

(C) Owned by the authors, published by EDP Sciences, 2012

\title{
Hyperfragments from light 1p-shell nuclei
}

\author{
L. Majling ${ }^{1,2, a}$, O. Majlingová ${ }^{3}$, V.A. Kuz' $\min ^{2}$, and T.V. Tetereva ${ }^{4}$ \\ 1 Nuclear Physics Institute, Academy of Sciences, Řež, Czech Republic \\ 2 Bogoliubov Laboratory of Theoretical Physics, JINR, Dubna, Russia \\ 3 Czech Technical University, Prague, Czech Republic \\ 4 Skobeltsyn Institute of Nuclear Physics, Lomonosov Moscow State University, Moscow, Russia
}

\begin{abstract}
Our contribution is inspired by the possibility to identify hyperfragments in the reaction $\left(e, e^{\prime} K^{+}\right)$. In the near future two experiments at JLab and Mainz will provide a systematic study of the fragments from the primary $p$-shell hypernuclei.
\end{abstract}

\section{Introduction - Hypernuclei}

Hypernuclei are unique in their potential of improving knowledge on strange particle - nucleus interaction which is essential for a more general description of the baryon-baryon (BB) interaction [1]. Strangeness physics is adding a new degree of freedom to our understanding of hadrons, their structure and their interactions within the fundamental $\mathrm{SU}(3)_{\text {flavor }}$. Promising candidate for self-consistent description of the BB interaction is chiral effective field theory $(\chi \mathrm{EFT})$. The effect of strange quarks in nuclear matter is tested through determination of the properties of light hypernuclei. In the recent years we see a "renaissance" of the hypernuclear physics in Europe [2]. Hypernuclear physics was a part of the 7th Framework Programme (FP7) of European Commission Hadron Physics 2 (now 3): Study of Strongly Interacting Matter. The working programme SPHERE (Strange Particles in Hadronic Environment Research in Europe) coordinates the studies of hypernuclei at Frascati, Mainz and GSI. The network tightens the relations among the various experimental and theoretical research groups in Europe (Germany, Italy, Spain, Czech Republic, Poland, Israel) and includes also teams working at CEBAF@J-Lab and J-PARC@ Tokai. There is also a close cooperation with Network LEANNIS (Low Energy Antikaon Nucleon and Nuclei Interaction Studies).

Hypernuclei are bridging the gap between physics of elementary particles and traditional nuclear spectroscopy. The JINR actually links these two branches. Here was born Podgoretskii's ingenious idea to use strangeness-exchange reaction $\left(K^{-}, \pi^{-}\right)$for production primary hypernuclei [3]. Since then hypernuclear spectroscopy is a standard constituent of intermediate-energy nuclear physics, solving the problems of interaction of variety of elementary particles $(e, \mu, \pi, K \ldots)$ with nuclei.

An example of the effective adoption of nuclear techniques is the proposal of Polikanov to measure the lifetime of hypernuclei using the delayed fission of heavy hyperfragments produced in the annihilation of $\bar{p}$ on Uranium [4].

\footnotetext{
a e-mail: majling@ujf.cas.cz
}

In BLTP were also born some interesting proposals: - to study hypernuclei with neutron halo [5];

- to study delayed clusters accompanying non-mesonic weak decay of some light hypernuclei (as delayed particles in $\beta$ decay) [6];

- to identify exotic hypernuclei ${ }_{\Lambda}^{6} \mathrm{H}$ and ${ }_{\Lambda}^{8} \mathrm{H}$ [7].

It is really our great pleasure to inform you that FINUDA Collaboration already published paper: Evidence for heavy hyperhydrogen ${ }_{\Lambda}^{6} \mathrm{H}[8]$.

The JINR hypernuclear physicists presented their current plan in Ref. [9].

\section{Motivation}

Our contribution is inspired by the possibility to identify hyperfragments in the reaction $\left(e, e^{\prime} K^{+}\right)$. In the near future two experiments at JLab [10] and Mainz [11] will provide a systematic study of the fragments from the primary $p$ shell hypernuclei. It is an important extension of the very successful experiments investigated discrete part of hypernuclear spectra.

As a first step we analyze the decay of the components of Strange Analogue States (SAS) $\left(p_{\Lambda} p^{-1}, s_{\Lambda} s^{-1}\right)$ for hypernuclei $A \leq 9$ using Translation Invariant Shell Model (TISM) $[12,13]$. The attention is focused on the impact of target's nuclear structure on the population of concrete decay channels.

\subsection{Excitation spectra}

In spectra of light hypernuclei produced by strangeness exchange reaction $\left(K_{\text {in flight }}^{-}, \pi^{-}\right)[14,15]$ we can see well defined unbound states with sufficiently narrow decay width. A nice example is hypernucleus ${ }_{\Lambda}^{6} \mathrm{Li}$, see figure 1 . In Ref. [16] we have discussed hypernucleus ${ }_{\Lambda}^{6} \mathrm{Li}$ in terms of an extended supermultiplet scheme which combines the $1 \mathrm{~s}$ and $1 p$ orbitals, and classifies the nuclear and hypernuclear states by Young tableaux $[f]$. The lower state has symmetry [41] for its nuclear core, so its break-up to ${ }_{\Lambda}^{5} \mathrm{He}+p$ or to ${ }^{5} \mathrm{Li}+\Lambda$ is allowed both energetically and by supermultiplet 


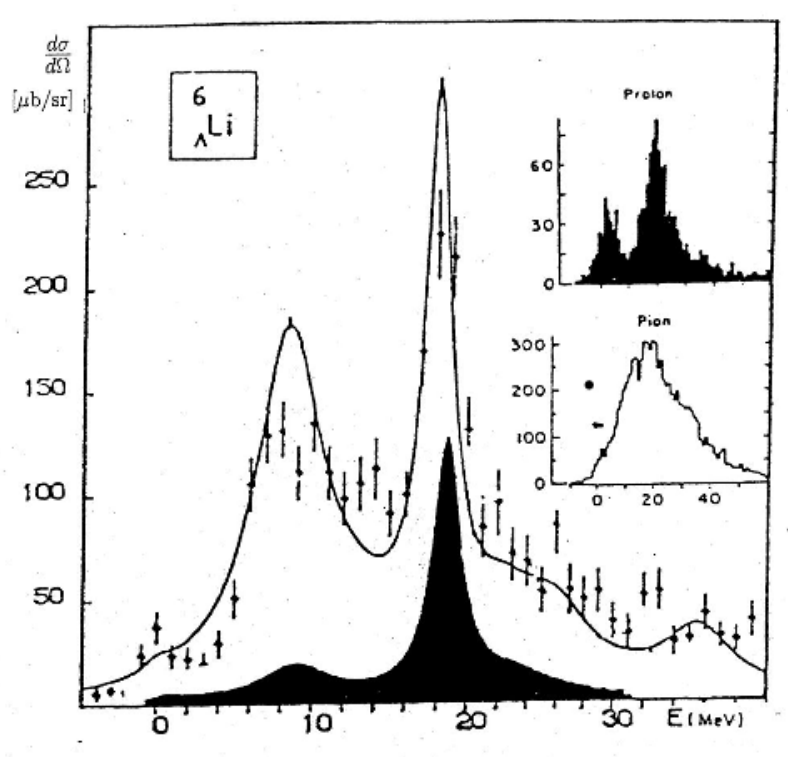

Fig. 1. The $\left(K^{-}, \pi^{-}\right)$spectrum of the ${ }_{\Lambda}^{6} \mathrm{Li}$ production [14]

symmetry. The upper state has symmetry [32] for its nuclear core so that its decay to ${ }^{5} \mathrm{Li}+\Lambda$ or ${ }_{\Lambda}^{5} \mathrm{He}+p$, is forbidden by the selection rules for the supermultiplet symmetry.

\subsection{Gamma's from hyperfragment}

The experiment ${ }^{10} \mathrm{~B}\left(K^{-}, \pi^{-} \gamma\right)$ observed $\gamma$ rays from ${ }_{\Lambda}^{7} \mathrm{Li}$ produced as hyperfragments from highly excited states of ${ }_{\Lambda}^{10} \mathrm{~B}$ [17].

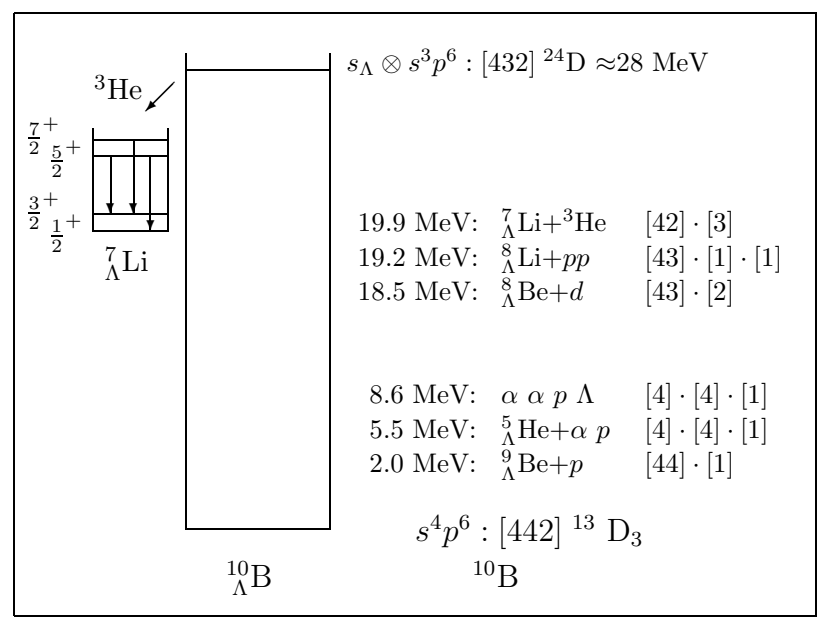

Again, supermultiplet symmetry forbids a decay through the channels ${ }_{\Lambda}^{9} \mathrm{Be}+p$ and ${ }_{\Lambda}^{5} \mathrm{He}+\alpha+p$ because they correspond to $[f]=[44]$.

\subsection{Proposal}

The Proposal [10] intends to use high precision monochromatic $\pi^{-}$'s from the unique two-body mesonic weak decay of hypernuclei to investigate light $\Lambda$-hypernuclei with variety of $(Z, A)$ combinations through identification of hyperfragments from strongly produced hypernuclear continuum (quasi free production) in $\left(e, e^{\prime} K^{+}\right)$electro-production.
A collection of hypernuclei: neutron-rich hypernuclei, neutron drip line hypernuclei (including ${ }_{\Lambda}^{6} \mathrm{H}$ and ${ }_{\Lambda}^{8} \mathrm{H}$ ), and mirror pairs of hypernuclei can be observed. The experiment will take a phase approach for the three different targets: ${ }^{7} \mathrm{Li},{ }^{9} \mathrm{Be}$, and ${ }^{12} \mathrm{C}$.

\section{Model}

It is a great challenge to work out the model for description fragment's emission from hypernuclear resonances. Here, we follow Ed Hungerford suggestion: "As our experimental and theoretical tools are sharpened, we proceed to build structure on top of foundations previously constructed. Much of what we do was anticipated by our predecessors."

\subsection{Strangeness Analogue State}

First of all we use a notion of Strangeness Analogue State (SAS) introduced by Kerman and Lipkin. It combines a simple theoretical structure with a source of rich and easily analyzed data of resonances [18]. Thus these states give us the "best of both world": the easily analyzed structure of nuclear ground state together with the rich data of continuum states.

For $1 p$-shell hypernuclei SAS do belong to the $1 \hbar \omega$ excitation band. The latter may be split into three groups, where the single-particle energies differ significantly.

\begin{tabular}{lllll}
\hline & & & \multicolumn{1}{c}{ decay } \\
\hline $1 \hbar \omega_{(\Lambda)}$ & $\left(\varepsilon_{p_{\Lambda}}\right)$ & $p^{-1} p_{\Lambda}$ & & $\Lambda$ \\
$1 \hbar \omega_{N}$ & $\left(\varepsilon_{d}\right)$ & & $p^{-1} d s_{\Lambda}$ & $N$ \\
$1 \hbar \omega_{N}$ & $\left(\varepsilon_{s^{-1}}\right)$ & $\underbrace{}_{\text {GDR } \otimes s_{\Lambda}} s^{-1} p s_{\Lambda}$ & ${ }_{\Lambda}^{4} \mathrm{H}, t$
\end{tabular}

This situation reminds "configurational splitting" of Giant Dipole Resonance [19].

Explicit expression for components of SAS in our cases is as follows :

\begin{tabular}{lcll}
\hline target & $n_{f}$ & \\
\hline${ }^{6} \mathrm{Li}: \mid s^{4} p^{2}[42](20)>$ & $\frac{4}{9}$ & $\frac{9}{10}$ & $\Phi_{1}[41] \otimes p_{\Lambda}$ \\
& $\frac{1}{10}$ & $\Phi_{2}[41] \otimes s_{\Lambda}$ \\
& $\frac{5}{9}$ & 1 & $\Phi_{2}[32] \otimes s_{\Lambda}$ \\
& & & \\
& $\frac{9}{14}$ & $\frac{7}{9}$ & $\Phi_{2}[42] \otimes p_{\Lambda}$ \\
& & $\frac{2}{9}$ & $\Phi_{3}[42] \otimes s_{\Lambda}$ \\
& $\frac{5}{14}$ & 1 & $\Phi_{3}[33] \otimes s_{\Lambda}$ \\
${ }^{7} \mathrm{Li}: \mid s^{4} p^{3}[43](30)>$ & & \\
& $\frac{1}{6}$ & $\frac{15}{16}$ & $\Phi_{4}[44] \otimes p_{\Lambda}$ \\
& $\frac{1}{16}$ & $\Phi_{5}[44] \otimes s_{\Lambda}$ \\
& $\frac{5}{6}$ & $\frac{9}{16}$ & $\Phi_{4}[431] \otimes p_{\Lambda}$ \\
& $\frac{7}{16} p^{5}[441](31)$ & $\Phi_{5}[431] \otimes s_{\Lambda}$
\end{tabular}

\subsection{TISM CFP}

The key ingredients in the shell model are coefficients of fractional parentage (CFP) - coefficients in decomposition 
of antisymmetrized wave function. For TISM we have :

$$
\begin{aligned}
\Phi_{k}^{(A)}[f](\lambda \mu)=\sum_{f_{1}, f_{2}} \sum_{k_{1}, k_{2}, v} & \\
\sqrt{\frac{n f_{1} \cdot n f_{2}}{n f}} & \Phi_{k_{1}}^{\left(A_{1}\right)}\left[f_{1}\right](\lambda \mu)_{1} \\
& \Phi_{k_{2}}^{\left(A_{2}\right)}\left[f_{2}\right](\lambda \mu)_{2} \\
& \varphi_{v}\left(R_{1}-R_{2}\right)
\end{aligned}
$$

Wave functions for "simplest" clusters $(d, t, \alpha)$ are $\mid s^{k}>\equiv$ $\Phi_{0}^{(k)}[k](00)$. For heavy Hydrogen isotopes we take:

$$
\begin{aligned}
& \mid s^{3} p:[31]>\equiv \Phi_{1}^{(4)}[31]\left({ }^{4} \mathrm{H}\right) \\
& \mid s^{3} p^{2}:[32]>\equiv \Phi_{2}^{(5)}[32]\left({ }^{5} \mathrm{H}\right) .
\end{aligned}
$$

Spectroscopic amplitude for hyperfragment emission

$$
{ }_{\Lambda}^{A} Z \rightarrow{ }_{\Lambda}^{A_{1}} Z_{1}+{ }^{A_{2}} Z_{2}
$$

is multiplied by factor $\frac{A+1}{A} \cdot \frac{A_{1}}{A_{1}+1}$ reflecting transformation of Jacobi coordinates .

\section{Predictions}

\subsection{Hyperfragments from ${ }_{\Lambda}^{6} \mathrm{He}$}

\begin{tabular}{|c|c|c|c|c|c|}
\hline$E_{\mathrm{thr}}$ & $\mathrm{X}+\mathrm{HF}$ & {$\left[f_{i}\right]\left[f_{k}\right]$} & CFP & CG J & SA \\
\hline 2.8 & $n+{ }_{\Lambda}^{6} \mathrm{He}$ & {$[1][41]$} & 0 & & 0 \\
\hline 3.1 & $2 n+{ }_{\Lambda}^{5} \mathrm{He}$ & {$[1][1][4]$} & 0 & & 0 \\
\hline 5.2 & $\Lambda+{ }^{6} \mathrm{He}$ & [42] & 0 & & 0 \\
\hline 15.5 & $" t "+{ }_{\Lambda}^{4} \mathrm{H}$ & [3][3] & $\frac{1}{5}$ & $1 \frac{8}{7} \frac{4}{5}$ & 0.183 \\
\hline 21.1 & $3 n+{ }_{\Lambda}^{4} \mathrm{He}$ & {$[1][1][1][3]$} & ] $\frac{1}{5}$ & 0 & 0 \\
\hline $22.1^{*}$ & “d" $+{ }_{\Lambda}^{5} \mathrm{H}$ & {$[2][31]$} & $\frac{13}{240}$ & $1 \frac{8}{7} \frac{5}{6}$ & 0.051 \\
\hline 23.7 & $" t n "+{ }_{\Lambda}^{3} \mathrm{H}$ & {$[3][1][2]$} & $\frac{1}{4}$ & $1 \frac{8}{7} \frac{3}{4}$ & 0.214 \\
\hline 23.8 & $p+{ }_{\Lambda}^{6} \mathbf{H}$ & {$[1][32]$} & $\frac{4}{27}$ & $\begin{array}{ll}\frac{3}{4} & \quad 8 \\
7 & \frac{6}{7}\end{array}$ & 0.109 \\
\hline
\end{tabular}

Spectroscopic amplitudes (SA) for decay of $s_{\Lambda} \otimes \Phi_{2}^{(5)}[32]^{24} S$

\begin{tabular}{rllc}
\hline$E_{\mathrm{thr}}$ & Decay & {$\left[f_{i}\right]\left[f_{k}\right]$} & $\mathrm{SA}$ \\
\hline 0.17 & ${ }_{\Lambda}^{5} \mathrm{He}+n$ & {$[4][1]$} & 0 \\
4.18 & ${ }^{5} \mathrm{He}+\Lambda$ & {$[41]$} & 0 \\
18.84 & ${ }_{\Lambda}^{4} \mathrm{H}+d$ & {$[3][2]$} & 0.153 \\
20.75 & ${ }_{\Lambda}^{3} \mathrm{H}+t$ & {$[2][3]$} & 0.352 \\
\hline
\end{tabular}

\subsection{Hyperfragments from ${ }_{\Lambda}^{7} \mathrm{He}$}

Spectroscopic amplitudes for decay of $s_{\Lambda} \otimes \Phi_{3}^{(6)}[33]^{33} \mathrm{P}$ are

Here, CG are Clebsch Gordan coefficients and J - TalmiMoshinski coefficient stemming from the transformation of different sets of Jacobi coordinates. The corresponding cluster configurations are

\begin{tabular}{cl}
\hline $\mathrm{X}+\mathrm{HF}$ & \\
\hline$n+{ }_{\Lambda}^{6} \mathrm{He}$ & $\Phi_{1}^{(5)}[41](10){ }^{22} \mathrm{P}$ \\
$2 n+{ }_{\Lambda}^{5} \mathrm{He}$ & $\Phi_{0}^{(4)}[4](00){ }^{11} \mathrm{~S}$ \\
$\Lambda+{ }^{6} \mathrm{He}$ & $\Phi_{2}^{(6)}[42](20){ }^{31} \mathrm{~S}$ \\
$“ t ”+{ }_{\Lambda}^{4} \mathrm{H}$ & $\Phi_{0}^{(3)}[3](00){ }^{22} \mathrm{~S}$ \\
$3 n+{ }_{\Lambda}^{4} \mathrm{He}$ & $\Phi_{0}^{(3)}[3](00){ }^{22} \mathrm{~S}$ \\
$“ d "+{ }_{\Lambda}^{5} \mathrm{H}$ & $\Phi_{1}^{(4)}[31](10){ }^{31} \mathrm{P}$ \\
$“ t n "+{ }_{\Lambda}^{3} \mathrm{H}$ & $\Phi_{0}^{(2)}[2](00){ }^{13} \mathrm{~S}$ \\
$p+{ }_{\Lambda}^{6} \mathrm{H}$ & $\Phi_{2}^{(5)}[32](20){ }^{42} \mathrm{~S}$ \\
\hline
\end{tabular}

\subsection{Hyperfragments from ${ }_{\Lambda}^{9} \mathrm{Li}$}

\begin{tabular}{|c|c|c|c|c|}
\cline { 3 - 5 } \multicolumn{2}{l|}{} & ${ }^{7} \mathrm{Li}$ & ${ }_{\Lambda}^{8} \mathrm{Li}$ & ${ }_{\Lambda}^{9} \mathbf{L i}$ \\
\hline $2 n$ & $n$ & $\Lambda$ \\
\hline${ }_{\Lambda}^{4} \mathrm{He}$ & ${ }^{5} \mathrm{He}$ & ${ }_{\Lambda}^{6} \mathrm{He}$ & ${ }_{\Lambda}^{7} \mathrm{He}$ & ${ }_{\Lambda}^{8} \mathrm{He}$ \\
$t n n$ & $t n$ & $t$ & $d$ & $p$ \\
31.5 & 9.9 & 9.7 & 13.0 & 13.8 \\
\hline${ }_{\Lambda}^{3} \mathrm{H}$ & ${ }_{\Lambda}^{4} \mathrm{H}$ & $\left({ }_{\Lambda}^{5} \mathrm{H}\right)$ & ${ }_{\Lambda}^{6} \mathrm{H}$ & $\left({ }_{\Lambda}^{7} \mathrm{H}\right)$ \\
${ }^{6} \mathrm{He}$ & ${ }^{5} \mathrm{He}$ & ${ }^{4} \mathrm{He}$ & ${ }^{3} \mathrm{He}$ & $2 p$ \\
18.2 & 11.8 & & 39.9 & \\
\hline
\end{tabular}

\begin{tabular}{rccll}
$\mathrm{E}_{\text {thr }}$ & \multicolumn{3}{c}{ decay } & {$\left[\mathrm{f}_{1}\right]\left[\mathrm{f}_{2}\right]$} \\
3.7 & $n$ & + & ${ }_{\Lambda}^{8} \mathrm{Li}$ & {$[1][43]$} \\
8.5 & ${ }^{8} \mathrm{Li}$ & + & $\Lambda$ & {$[431]$} \\
9.7 & $t$ & + & ${ }_{\Lambda}^{6} \mathrm{He}$ & {$[3][41]$} \\
9.9 & $t n$ & + & ${ }_{4}^{5} \mathrm{He}$ & {$[3][1][4]$} \\
11.8 & ${ }^{5} \mathrm{He}$ & + & ${ }_{\Lambda} \mathrm{H}$ & {$[41][3]$} \\
12.2 & $2 n$ & + & ${ }_{\Lambda}^{7} \mathrm{Li}$ & {$[1][1][42]$} \\
13.0 & $d$ & + & ${ }_{\Lambda}^{7} \mathrm{He}$ & {$[2][42]$} \\
13.8 & $p$ & + & ${ }_{\Lambda}^{8} \mathrm{He}$ & {$[1][421]$} \\
18.2 & ${ }^{6} \mathrm{He}$ & + & ${ }_{\Lambda}^{3} \mathrm{H}$ & {$[42][2]$} \\
31.5 & $t n n$ & + & ${ }_{1}^{4} \mathrm{He}$ & {$[3][1][1][3]$} \\
39.9 & ${ }^{3} \mathrm{He}$ & + & ${ }_{\Lambda}^{6} \mathrm{H}$ & {$[3][32]$}
\end{tabular}

\section{Summary}

We explore simple "toy model" to analyze cluster decay of primary hypernuclei. It is an extension of a very successful analysis of $\gamma$-transitions accomplished by Millener [20]. And it is complementary to transport model by Botvina $e t$ al. $[2,21]$. Our results may be useful in judicious choice of targets as paper by Dalitz and Gal [22] was at dawn of hypernuclear $\gamma$ spectroscopy.

\section{Acknowledgements}

L.M. wishes to thank organizers for their kind invitation to this highly stimulated conference, hospitality and financial 
support. Work of L.M. was supported by grant P203/12/2126 of the Grant Agency of the Czech Republic. It is also part of the European Union Initiative FP7, Study of Strongly Interacting Matter (HadronPhysics3, SPHERE network). Work of O.M. was supported by Project LA08002 of the Ministry of Education, Youth and Sport of the Czech Republic.

\section{References}

1. E. Botta, T. Bressani, G. Garbarini, Eur. Phys. J. A 48, $41(2012)$

2. J. Pochodzalla, Acta Phys. Polonica B 42, 833 (2011)

3. M.I. Podgoretskii, JETP 44, 695 (1963)

4. S. Polikanov, Nucl. Phys. A 478, 805c (1988)

5. L Majling, in Few-Body Problems in Physics. Proc. National Conference on Physics of Few-Body and Quark-Hadronic Systems, Kharkov, Ukraine, 1992, p. 345 (KFTI, Kharkov, 1994); Nucl. Phys. A 585, 211c (1995)

6. Yu. Batusov, et al., Phys. Elem. Part. At. Nucl. 36, 169 (2005);

L. Majling, V. Kuz'min, T. Tetereva, Phys. At. Nucl. 69, 838 (2006)

7. L. Majling, Š. Gmuca, Phys. At. Nucl. 70, 1611 (2007)

8. FINUDA Coll. and A. Gal, Phys. Rev. Lett. 108, 042501 (2012)

9. A.V. Averyanov, et al., Phys. At. Nucl. 71, 2101 (2008)

10. L. Tang, et al., Study of light hypernuclei by pionic decay. Proposal E-10-001, Jefferson Lab.

11. P. Auerbach, A1 Collaboration, Nucl. Phys. A 881, 187 (2012)

12. J. Žofka, et al., Sov. J. Part. Nucl. 22, 1292 (1991)

13. L. Majling, V.N. Fetisov, R.A. Eramzhyan, Sov. J. Part. Nucl. 28, 101 (1997)

14. R. Bertini, et al., Nucl. Phys. A 368, 365 (1981)

15. J.J. Szymanski, et al., Phys. Rev. C 43, 849 (1991)

16. L. Majling, et al., Phys. Lett. B 92, 256 (1980)

17. O. Hashimoto, H. Tamura, Progr. Part. Nucl. Phys. 57, 564 (2006)

18. H.J. Lipkin, Summer Study Meeting on Nuclear and Hypernuclear Physics with Kaon Beams, (BNL 18335, BNL, Upton, 1973), p. 147.

19. R. Eramzhyan, B. Ishkhanov, I. Kapitonov, V. Neudatchin, Phys. Rep. 136, 229 (1986)

20. D.J. Millener, Topics in Strangeness Nuclear Physics, Lecture Notes in Physics, 724 (Springer, Berlin Heidelberg, 2007), p. 31.

21. A.S. Botvina, et al., Nucl. Phys. A 881, 228 (2012)

22. R.H. Dalitz, A. Gal, Ann. Phys. 116, 167 (1978) 